\title{
A CUBESAT-READY PHASE SYNCHRONIZATION DIGITAL PAYLOAD FOR COHERENT DISTRIBUTED REMOTE SENSING MISSIONS
}

\author{
${ }^{1}$ J. Querol, ${ }^{1}$ J. C. Merlano-Duncan, ${ }^{1}$ L. Martinez-Marrero, ${ }^{1}$ J. Krivochiza, ${ }^{1}$ S. Kumar, ${ }^{1}$ N. Maturo, \\ ${ }^{2}$ A. Camps, ${ }^{1}$ S. Chatzinotas, ${ }^{1}$ B. Ottersten \\ ${ }^{1}$ Interdisciplinary Centre for Security Reliability and Trust (SnT), University of Luxembourg \\ ${ }^{2}$ Unidad María de Maeztu CommSensLab - Dept. of Signal Theory and Communications, Universitat \\ Politècnica de Catalunya-BarcelonaTech and IEEC/CTE-UPC \\ E-mail: \{jorge.querol, juan.duncan, liz.martinez-marrero, jevgenij.krivochiza, sumit.kumar, \\ nicola.maturo, symeon.chatzinotas, bjorn.ottersten\}@uni.lu; \\ camps@tsc.upc.edu
}

\begin{abstract}
Distributed antenna arrays, fractionated payloads and cooperative platforms can provide unprecedented performance in the next generation of spaceborne communications and remote sensing systems. Remote phase synchronization of physically separated oscillators is the first step towards a coherent operation of distributed systems. This work shows the preliminary results of a TDD remote phase synchronization algorithm with a master-follower architecture. Herein, we describe the implementation and validation of the proposed algorithm. The implementation has been conducted in a Cubesat-ready software defined radio and validated at the end-to-end satellite communications testbed available at the University of Luxembourg.
\end{abstract}

Index Terms - Synchronization, multi-static remote sensing systems, distributed beamforming, software defined radio.

\section{INTRODUCTION}

Distributed payloads, decentralized systems, cooperative platforms, and collaborative beamforming are crucial elements enabling the next generation of multi-static remote sensing systems. For example, future microwave radiometers could benefit from an increased aperture size conformed by antenna elements in a flying formation operating coherently as a distributed antenna array [1],[2].

Time and frequency, and phase synchronization is a key element in the operation of distributed remote sensing and communications infrastructures. The current technology already relies on precise synchronization realized by distributing a common clock and local oscillator (LO) references through very stable wired connections.

However, the synchronization requirements become even more challenging when distributed synchronization need to be achieved wirelessly among physically separated nodes with independent free running LO references. The main difficulties found in these scenarios, on top of the movement of the nodes, are fading, multi-path and non-reciprocity of the channel, which makes a practical implementation of multistatic systems highly challenging in cluttered or indoor radio environments.

Overcoming the implementation challenges of a reliable, accurate and efficient remote wireless LO synchronization will enable a new plethora of applications in remote sensing. The cooperative operation can be either an active or passive remote sensing instrument such as distributed synthetic aperture radiometer or radar benefitting from longer baselines between the antenna elements and overcoming the physical challenges of building a single unit satellite with considerable dimensions. Another application can be a distributed interference detector using Angle-Of-Arrival (AOA) estimation between the synchronized receiving payload nodes.

This paper is focused on the implementation challenges of a real-time system for remote phase synchronization with a master-follower topology. The work is restricted to the implementation of two remote phase synchronization nodes in a Cubesat-ready Software Defined Radio (SDR) payload for feasibility study and demonstration purposes. The algorithm has been already described and tested with ground equipment in [3],[4]. It is assumed that the nodes to be synchronized are either orbiting, flying or hovering, and they are part of a distributed system performing a cooperative coherent operation. Temporal synchronization is also of paramount importance for distributed systems, but it is not considered in this work. An example of distributed time synchronization can be found in [5].

\section{ALGORITHM DESCRIPTION}

Herein, we assume a master-follower architecture works as a remote Digital Phase Locked Loop (DPLL). The master node transmits a reference signal to sounds and measures the phase 
error introduced by the wireless channel between the master and the follower node. The function of the follower node is to track the phase of the reference signal and to retransmit it back to the master node. The master node closes the loop by estimating the phase error between the received and generated signals. The main rationale behind the phase synchronization is that, once the master and follower nodes are in phase, the estimation of the phase difference will be equal to twice the phase introduced by the round-trip reciprocal channel. Based on this principle, the master precompensates the phase introduced by the channel in order to cancellate the phase error between both nodes and, therefore, to achieve the remote phase synchronization.

The reference signal used for the phase estimation may be either a dedicated signal or some opportunistic pilot signal from a communications protocol such as DVB-S2(X), widely used in satellite communications, or 3GPP, such as 5G-NR Phase-Tracking Reference Signal (PTRS) designed specifically for phase noise compensation [6]. In any case, a number of phase estimation algorithms (e.g. R\&B, Fitz, $M \& M)$ are available in the literature to effectively estimate the carrier phase out of the pilot reference signal.

In the implementation of the DPLL architecture, both nodes should use the same carrier frequency for the (re)transmission of their respective signals. Otherwise, the loop will not achieve a perfect synchronization since the drifts in the respective RF chains will be different and unpredictable. A solution based on In-Band Full-Duplex (IBFD) techniques would solve this problem, but it leads to a non-trivial hardware implementation. There are two practical alternatives: Frequency Division Duplex (FDD) and Time Division Duplex (TDD).

On one hand, the FDD scheme must use twin carriers symmetric with respect to a center frequency $f_{c}$, where the master and the follower nodes transmit two identic reference signals at frequencies $f_{c} \pm f_{m}$ and $f_{c} \pm f_{s}$ respectively. The use of symmetric carriers guarantees that the undesired phase errors introduced by the RF components at different frequencies are averaged out with respect to the central frequency $f_{c}$.
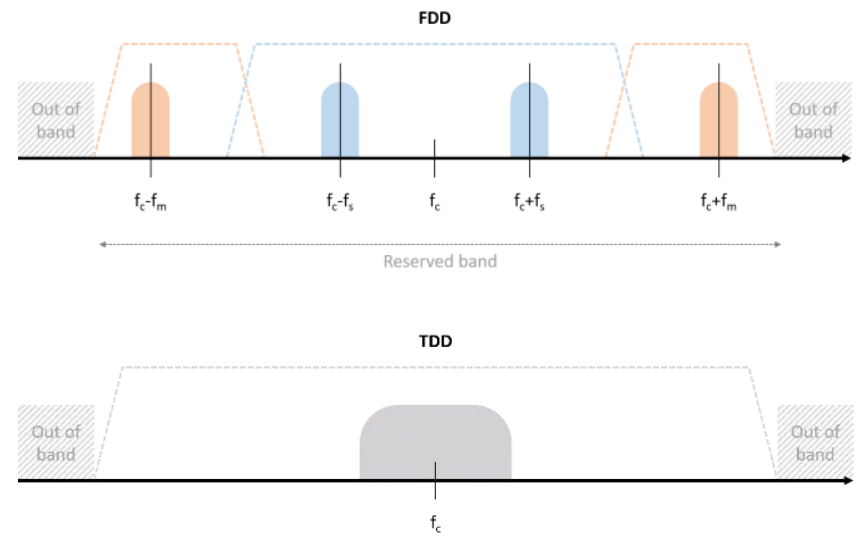

Fig. 1: FDD and TDD configuration frequency plan.
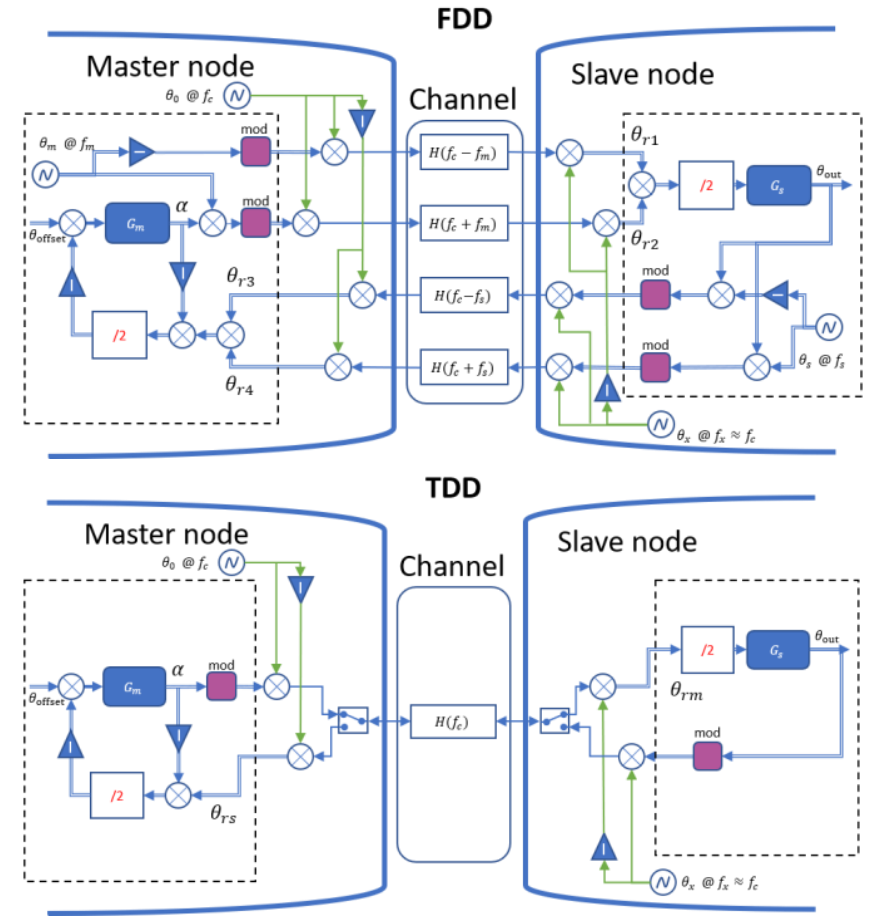

Fig. 2: Diagram of the proposed master-follower phase synchronization algorithm for both FDD and TDD schemes.

On the other hand, the TDD scheme allows reusing the same frequency band for the bidirectional synchronization, thus ensuring the reciprocity of the system. However, the synchronization may be degraded due to the increased roundtrip latency and the discontinuous sensing as compared to the FDD scheme.

Fig. 1 shows a diagram of the proposed algorithm for both FDD and TDD schemes.

\section{SDR IMPLEMENTATION}

The proposed remote synchronization algorithm has been implemented in a space-qualified Software Defined Radio (SDR) from GOMSPACE. This SDR device is composed by a Xilinx Zynq 7030 FPGA connected to a 2x2 AD9361 transceiver. The center frequency has been tuned to $2245 \mathrm{MHz}$, corresponding to the center of the reserved band for satellite-to-satellite operation at S-band $(2200 \mathrm{MHz}-$ $2290 \mathrm{MHz}$ ). The maximum sampling frequency of the SDR is $54 \mathrm{MHz}$, which is given by the AD9361 transceiver.

The TDD scheme is preferable for this implementation since the hardware implementation of the FDD scheme in such a narrow operating band $(54 \mathrm{MHz})$ will led to an improper rejection of the duplexing filters. However, this does not mean that the FDD scheme is not feasible, but it will require hardware modifications to accommodate the four carriers in a larger bandwidth.

The TDD scheme proposed in Fig. 2 has been implemented in the Zynq 7030 FPGA by using a simplified 
version of the PTRS proposed in the 5G standard. Each node has assigned a subframe or slot of $1 \mathrm{~ms}$ per transmission with a configurable phase-tracking pilot time density of the $100 \%$ as in [7].

\section{TESTBED DESCRIPTION}

The satellite communications testbed developed by the University of Luxembourg is composed by a channel emulator and several SDR-based end-to-end transceivers compliant with different communication standards (e.g. DVB and 3GPP). Fig. 3 shows the testbed comprising of a spectrum analyzer, the Cubesat SDRs used for the implementation of the remote synchronization payload, the channel emulator board and its CPU controlled, and several NI USRP SDR devices for the transceiver implementation or generalpurpose signal generation. All the elements in the setup are interconnected using coaxial cables working at an L-/S-band.

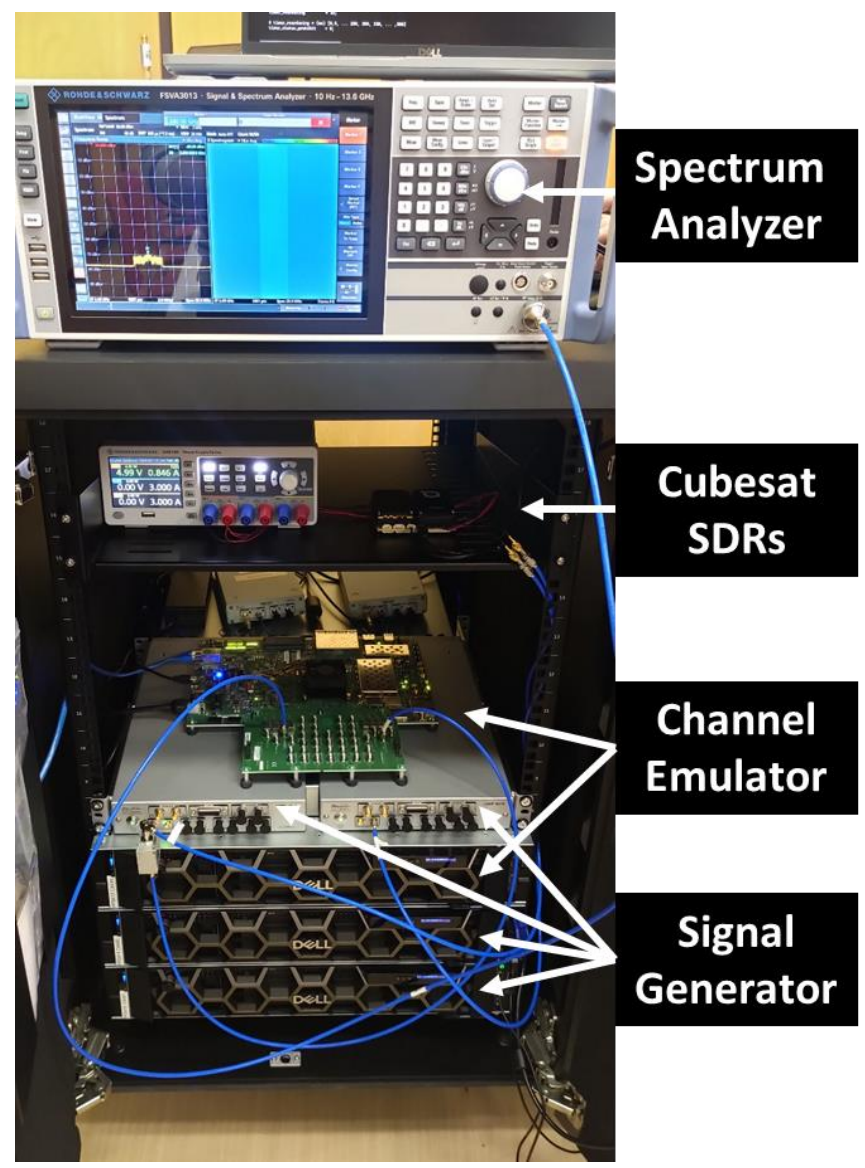

Fig. 3: End-to-end satellite communications testbed available at the University of Luxembourg.

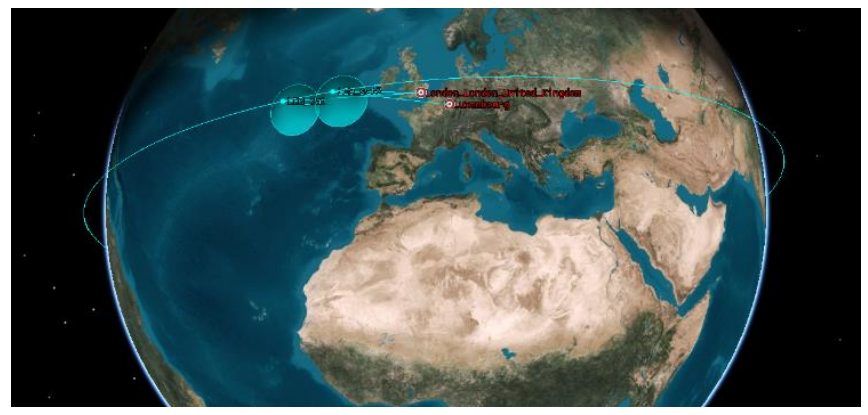

Fig. 4: Sample LEO orbit with two following Cubesats designed in STK $(500 \mathrm{~km}$ altitude and 52 degrees of inclination).

The channel emulator is implemented using a Zynq UltraScale+ RFSoC with up to eight direct RF sampling ADCs and DACs. The channel emulator can reproduce dynamically the channel conditions of the LEO scenario considering not just the static geometry, but also the antenna pattern and the attitude of the Cubesat, the operating frequency band and SNR of the targeted application, or the dynamic effects of the scenario such as differential propagation delay and Doppler effect. This is achieved by using the AGI STK software, which has been integrated into the channel emulator architecture. Fig. 4 shows a sample scenario with two satellites in LEO orbit with a nadir-looking S-band patch antenna.

\section{RESULTS}

The implementation of the 5G TDD transceiver has been simplified to the bare minimum. Both control and broadcast channels have not been implemented. Therefore, the resource allocation is assumed constant and known by both gNB and UE sides, and the initial synchronization procedure is assumed to be already performed.

In this paper, the subcarrier spacing is set to $15 \mathrm{kHz}$ and the total bandwidth to $5 \mathrm{MHz}$ (i.e. 300 carriers) to minimize the amount of resources needed for the demonstrator. Fig. 5 shows the TDD symbol-based scheduling for each communication slot of $1 \mathrm{~ms}$. From all the 14 OFDM symbols available in each slot, 6 are assigned to the downlink (DL), 6 are assigned to the uplink (UL) and 2 are guard symbols. The allocation of the PTRS signals and the rest of the reference signals (CSIRS and DMRS) has been done symmetrically. The pattern shown in Fig. 5 is then repeated every $1 \mathrm{~ms}$. Using the time dense PTRS, both master and follower can track the phase of the channel and report back to the other node as shown in Fig. 2.

In this case, the channel has been simulated to be constant for simplicity, with a phase of 45 degrees with respect to the carrier. The main goal of this test is to show that the remote synchronization DPLL is correctly working. The RF setup has been set to be symmetrical, in the sense that equal SNR are expected to be measured at both nodes. 


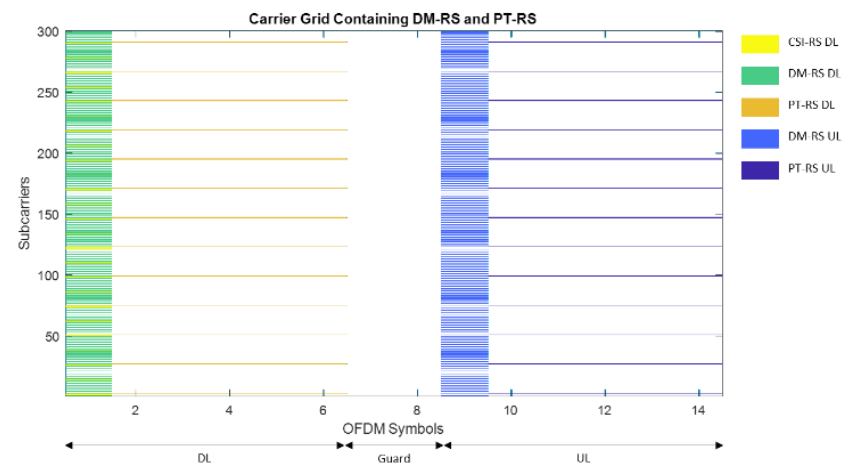

Fig. 5: TDD Symbol Based Scheduling with symmetric DL-UL pattern.

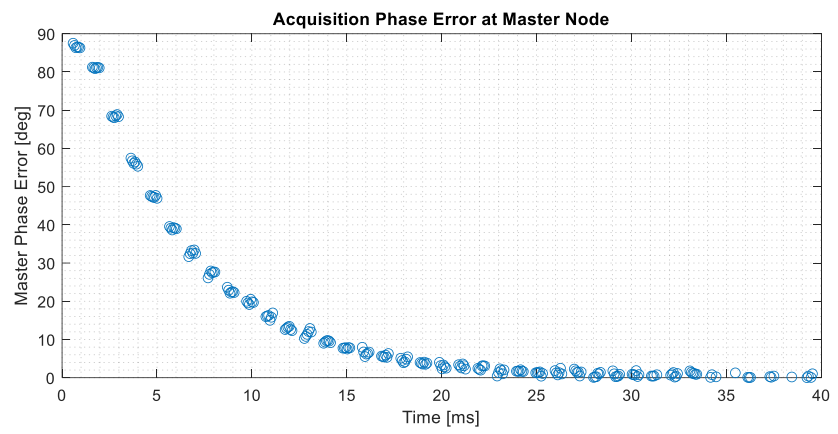

Fig. 6: Acquisition phase error at the Master Node.

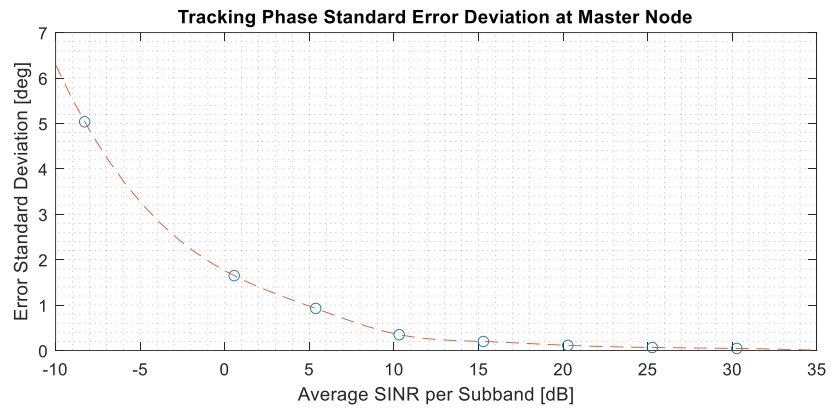

Fig. 7: Tracking error (standard deviation) at the Master Node as a function of the SINR per subband.

Fig. 6 shows the synchronization algorithm in the acquisition mode. The DPLL minimizes the phase error estimated at the Master node. The algorithm starts at 90 degrees because of the two-way 45 degrees channel. Note the $1 \mathrm{~ms}$ distance between clustered phase measurements. In this case, the DPLL bandwidth is not optimized as a function of the SNR since the main objective is to demonstrate the operation concept.

Fig. 7 shows the standard deviation of the phase error measured by the Master node as a function of the SINR. Note that below $-8 \mathrm{~dB}$, data cannot be exchanged between the nodes although the DPLL can be still locked. As compared to the FDD approach in [3], the TDD approach shows similar results if a regular pilot schedule is set, for example, at $1 \mathrm{~ms}$.

\section{CONCLUSIONS}

This paper shows the design, implementation, and results of a TDD remote synchronization scheme based on 5G NR reference signals. The proposed approach uses a fixed and regular grid of pilot signals to estimate, track and compensate the phase of the channel. The regularity of the pilots allows to get a performance equivalent to the FDD scheme with a simplified front-end architecture. Further research needs to address the design of a such a scheduler or a modification of the standard to accommodate a continuous transmission of pilots supporting the remote synchronization operation.

\section{ACKNOWLEDGMENTS}

This work was supported by the Fond National de la Recherche Luxembourg, under the CORE project Cognitive Cohesive Networks of Distributed Units for Active and Passive Space Applications (COHESAT), FNR/C17/IS/11689919/COHESAT/Merlano Duncan.

\section{REFERENCES}

[1] A. K. S. El Maghraby, A. Grubisic, C. Colombo, and A. Tatnall, "A Hexagonal Pseudo-polar FFT for FormationFlying Interferometric Radiometry," IEEE Geosci. Remote Sens. Lett., vol. 16, no. 3, pp. 432-436, Mar. 2019, doi: 10.1109/LGRS.2018.2876022.

[2] W. J. Blackwell, "Technology Evolution to Enable HighPerformance Cubesat Radiometry Missions," IGARSS 2019 - 2019 IEEE Int. Geosci. Remote Sens. Symp., pp. 5078-5081, Jul. 2019, doi: 10.1109/IGARSS.2019.8897993.

[3] J. C. M. Duncan et al., "SDR Implementation of a Testbed for Synchronization of Coherent Distributed Remote Sensing Systems" IGARSS 2020 - 2020 IEEE International Geoscience and Remote Sensing Symposium, Honolulu, Hawai, US, 2020.

[4] J. C. M. Duncan et al., "A Remote Carrier Synchronization Technique for Coherent Distributed Remote Sensing Systems," in IEEE Journal of Selected Topics in Applied Earth Observations and Remote Sensing, doi: 10.1109/JSTARS.2020.3046776.

[5] S. Prager, M. S. Haynes and M. Moghaddam, "Wireless Subnanosecond RF Synchronization for Distributed Ultrawideband Software-Defined Radar Networks," in IEEE Transactions on Microwave Theory and Techniques, vol. 68 , no. 11, pp. 4787-4804, Nov. 2020, doi: 10.1109/TMTT.2020.3014876.

[6] E. Dahlman, J. Skold, 5G NR: The Next Generation Wireless Access Technology. Elsevier Science, 2018, doi: 10.1016/C2017-0-01347-2.

[7] Y. Qi, M. Hunukumbure, H. Nam, H. Yoo and S. Amuru, "On the Phase Tracking Reference Signal (PT-RS) Design for 5G New Radio (NR)," 2018 IEEE 88th Vehicular Technology Conference (VTC-Fall), Chicago, IL, USA, 2018, pp. 1-5, doi: 10.1109/VTCFall.2018.8690852. 